\title{
Diferencias de especie en las concentraciones plasmáticas y tisulares de florfenicol entre ovinos y conejos
}

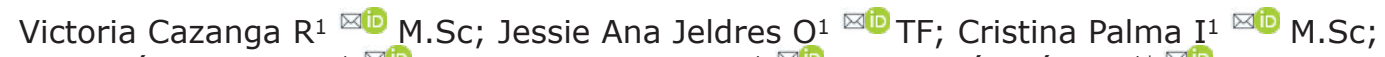

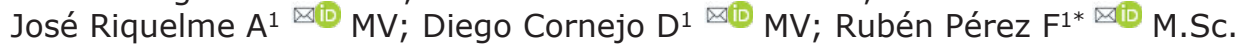

${ }^{1}$ Universidad de Concepción, Facultad de Ciencias Veterinarias, Departamento de Ciencias Clínicas, Laboratorio de Farmacología, Avenida Vicente Méndez 595, Chillán, Chile.

*Correspondencia: rubperez@udec.cl

Recibido: Junio 2019; Aceptado: Septiembre 2019; Publicado: Diciembre 2019.

\section{RESUMEN}

Objetivo. Comparar las concentraciones plasmáticas y tisulares de florfenicol (FFC) y su metabolito florfenicol amina (FFC-a) entre ovinos y conejos, posterior a la administración intramuscular de $20 \mathrm{mg} / \mathrm{kg}$ de FFC. Materiales y métodos. Cinco ovinos Suffolk Down y seis conejos Neozelandés fueron utilizados en el estudio. Se colectaron muestras de sangre, previo a la administración de FFC, y a las $0.25,0.5,1,1.5,2,3$ y 4 horas posteriores al tratamiento. A las 4 horas posteriores al tratamiento, a los animales se les aplicó la eutanasia. Las concentraciones plasmáticas y tisulares de FFC y FFC-a fueron determinadas mediante HPLC. Resultados. Las concentraciones plasmáticas máximas, tasa de absorción, vida media de absorción, tasa de distribución y área bajo la curva de FFC, fueron significativamente mayores en conejos respecto a los ovinos. Asimismo, para FFC-a, las concentraciones plasmáticas máximas y área bajo la curva de concentraciones plasmáticas en el tiempo fueron significativamente mayores en conejos respecto a los ovinos. La proporción de metabolito fue mayor en conejos $(12.7 \pm 3.07 \%)$ en comparación con ovinos $(3.99 \pm 0.87 \%)(p<0.05)$, al igual que las concentraciones tisulares de FFC y FFC-a. Conclusiones. Se observaron diferencias significativas en la farmacocinética y concentraciones tisulares de FFC y FFC-a entre estas dos especies. La mayor concentración de FFC-a en conejos indica un mayor nivel de metabolismo de FFC, respecto a los ovinos. Esto es importante de considerar al momento de establecer dosificaciones y frecuencia de administración de FFC en conejos.

Palabras clave: Antibióticos, cromatografía, farmacocinética, metabolismo (Fuente: MeSH).

\section{ABSTRACT}

Objective. The aim of this study was to compare tissue and plasma concentrations of florfenicol (FFC) and its metabolite florfenicol amine (FFC-a) between sheep and rabbits, after intramuscular administration of $20 \mathrm{mg} \mathrm{FFC} / \mathrm{kg}$. Materials and methods. Five Suffolk Down sheep and six New Zealand rabbits were used in this study. Blood samples were collected before FFC administration and at $0.25,0.5,1,1.5,2,3$ and 4 hours after treatment. At 4 hours after treatment, euthanasia was applied to animals. Plasma and tissue concentrations of FFC and FFC-a were determined by HPLC. Results. For FFC, maximum plasma concentrations, absorption rate, absorption half-life, distribution 
rate, and area under the plasma concentration-time curve were all found to be significantly higher in rabbits than in sheep. Similarly, for FFC-a, significantly higher maximum plasma concentrations and area under the concentration-time curve were observed in rabbits as compared to sheep. The metabolite ratio was higher in rabbits $(12.7 \pm 3.07 \%)$ compared to sheep $(3.99 \pm 0.87 \%)(p<0.05)$, as were the tissue concentrations of FFC and FFC-a. Conclusions. Significant differences in the pharmacokinetics and tissue concentrations of FFC, and its metabolite FFC-a, were observed between these two animal species. The higher concentrations of FFC-a in rabbits indicate a greater level of FFC metabolism as compared to sheep. This should be considered when establishing dosage and frequency of FFC administration for rabbits.

Keywords: Antibiotics, chromatography, metabolism, pharmacokinetics (Source: MeSH).

\section{INTRODUCCIÓN}

Florfenicol (FFC) es un antibiótico de amplio espectro, altamente efectivo para el control de infecciones del tracto respiratorio en bovinos y cerdos (1). FFC posee alta biodisponibilidad, buena penetración tisular, y rápida eliminación, atributos que lo hacen especialmente adecuado para ser usado en animales (1). FFC posee eficacia contra diversos patógenos Grampositivos y Gram-negativos como Pasteurella spp. (2), Staphylococcus aureus (1), Klebsiella pneumoniae (1), y Escherichia coli (2). Su uso ha sido aprobado en rumiantes y cerdos (3) y tiene el potencial de ser usado en especies menores como el conejo $(1,2)$.

FFC es altamente lipofílico (4) y se metaboliza en el hígado mediante el sistema citocromo P450 (CYP450) $(2,3,4,5)$. Diversos estudios han demostrado que posterior a su administración, FFC es transformado parcialmente a varios metabolitos, siendo florfenicol amina (FFC-a) el principal de los metabolitos derivados de FFC (6).

La farmacocinética de FFC ha sido descrita en pollos (4), cerdos (7), perros (8), conejos (9), ovinos (10), y ganado bovino $(11,12)$. Es sabido que la farmacocinética de fármacos en animales puede variar dependiendo de distintos factores, incluyendo variaciones entre especies (13).

Las enzimas del CYP450 juegan un rol crucial en el metabolismo de xenobióticos en las especies de mamíferos (14), convirtiendo varios fármacos en productos que pueden ser excretados del organismo. Este sistema se encuentra localizado principalmente en el hígado y está compuesto por varios subtipos enzimáticos, de los cuales CYP3A es una de las principales enzimas que participan del metabolismo de fármacos
$(2,14)$. Se sabe que existen diferencias en el metabolismo y excreción de fármacos entre conejos (monogástricos) y rumiantes como bovinos y ovinos $(15,16)$.

La farmacocinética comparativa de FFC ha sido descrita en camellos, ovejas y cabras (17), y también en aves como pollos, palomas y codornices (18). Sin embargo, de acuerdo a nuestro conocimiento no existen reportes disponibles que comparen concentraciones plasmáticas y tisulares de FFC y FFC-a entre ovinos y conejos. Ambas son especies de interés veterinario, y también representativas de rumiantes y monogástricos, respectivamente. Por ello son especies informativas que pueden ser usadas en estudios comparativos de las diferencias farmacocinéticas y de metabolismo de antibióticos. Por lo tanto, el objetivo de este estudio fue investigar si existen diferencias en las concentraciones plasmáticas y tisulares de FFC y FFC-a entre ovinos y conejos tratados con FFC vía intramuscular (IM).

\section{MATERIALES Y MÉTODOS}

\section{Animales de experimentación y ubicación} geográfica. Se utilizaron cinco ovinos adultos de la raza Suffolk Down, clínicamente sanos; machos castrados de 12 meses de edad, con un peso corporal (PC) entre $65 \pm 5 \mathrm{~kg}$. Los ovinos se mantuvieron estabulados en corrales colectivos, con cama de paja, alimentados con heno de alfalfa y un suplemento de concentrado $(300 \mathrm{~g} /$ ovino). Seis conejos Neozelandés clínicamente sanos, machos de 6 meses de edad entre $3.5 \pm$ $0.5 \mathrm{~kg} \mathrm{PC}$, fueron usados en este estudio. Los conejos se mantuvieron en jaulas separadas con 
acceso a bebedero automático. Se alimentaron con concentrado peletizado de alfalfa, y el agua se administró ad libitum en ambas especies.

Todos los animales fueron considerados clínicamente sanos y aptos para participar en el estudio después de realizar exámenes clínicos, parasitológicos y de bioquímica sanguínea. Los animales no tuvieron exposición previa a antibióticos, y no fueron administrados fármacos por un mes previo a los experimentos.

Este estudio se llevó a cabo en la Facultad de Ciencias Veterinarias, Universidad de Concepción, Chillán, Chile, en las dependencias del Hospital de grandes animales. Los procedimientos analíticos se realizaron en el Laboratorio de Farmacología, de la Facultad de Ciencias Veterinarias de la Universidad de Concepción.

Fármacos y reactivos. Los estándares analíticos de FFC (99.4\% pureza) y FFC-a (99.8\% pureza) fueron obtenidos de Sigma (Saint Louis, MO, USA), y Toronto Research Chemicals (North York, ON, Canadá), respectivamente. Una solución inyectable de FFC al 30\% (Nuflor $尺$ ) fue obtenida de Intervet Chile (bajo licencia de Schering Plough, Santé Animale ZA la Grindoliere-Segré France). Adicionalmente, la solución inyectable de FFC fue diluida en una solución constituida por $\mathrm{N}$-metil pirrolidona, polietilenglicol y propilenglicol (36:21:43, v/v) para obtener una concentración final de $60 \mathrm{mg} / \mathrm{mL}$. Esta dilución fue necesaria para poder obtener un volumen de inyección apropiado y para facilitar la inyección IM de $1 \mathrm{~mL}$ cada $3 \mathrm{~kg}$ PC en los conejos. También se observó que las características de densidad de la solución original impedían una administración adecuada y precisa de FFC en los conejos debido al pequeño volumen de inyección y a la alta densidad de la solución.

Todos los reactivos usados para extracción y procedimientos cromatográficos fueron grado cromatografía líquida de alta resolución (HPLC) (Merck, Darmstadt, Germany).

Diseño experimental. Los animales recibieron una inyección IM de FFC en una dosis de 20 $\mathrm{mg} / \mathrm{kg}$ PC, en el miembro posterior izquierdo. FFC fue inyectado a todos los animales a las 8.00 am para evitar variaciones en el ciclo día/ noche. En los ovinos se administró FFC al 30\% solución inyectable; los conejos recibieron una inyección de una solución de FFC al 6\%. El momento de la inyección fue considerado como tiempo cero (T0). Para el estudio farmacocinético se colectaron muestras de sangre previo al tratamiento con FFC y a las $0.25,0.5,1,1.5,2$, 3 , y 4 horas posteriores a su administración. A las 4 horas después del tratamiento, se aplicó la eutanasia a los animales con una sobredosis de anestésico, de acuerdo a las recomendaciones de las Guías de la AVMA para la Eutanasia de Animales (19). En los ovinos, la eutanasia se realizó a través de una inyección intravenosa (IV)

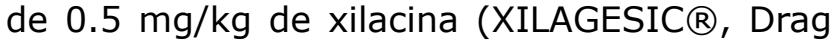
Pharma Chile Invetec S.A., Chile) y la inyección de solución de eutanasia (T-61ß, MSD Animal Health, bajo licencia de Intervet International B.V., Holland). Los conejos fueron eutanasiados con $4 \mathrm{mg} / \mathrm{kg}$ de xilacina mediante inyección IV, seguido por inyección de tiopental (OPET $®$, fabricado y distribuido por Pro-Vet S.A.).

Procedimientos de muestreo. Las muestras sanguíneas fueron obtenidas desde la vena yugular en ovinos y desde la vena marginal de la oreja en conejos ( $5 \mathrm{~mL}$ en ovinos y $1.5 \mathrm{~mL}$ en conejos). Las muestras fueron colectadas en tubos heparinizados y centrifugadas a $1372 \times g$ para obtener el plasma, el cual fue almacenado a $-18^{\circ} \mathrm{C}$. Cuatro horas posteriores al tratamiento, a ovinos y conejos se les aplicó el protocolo de eutanasia según procedimiento descrito anteriormente y se colectaron muestras de músculo, hígado, riñón, pulmón, bazo y cerebro, y fueron homogenizadas y almacenadas a $-18^{\circ} \mathrm{C}$ hasta su análisis en el Laboratorio de Farmacología.

Procedimientos analíticos. Las muestras de sangre y tejidos fueron procesadas para su análisis mediante cromatografía líquida de alto rendimiento (HPLC). Las concentraciones de fármaco fueron determinadas a partir del área de los picos cromatográficos y mediante curvas de calibración. Las curvas de calibración fueron obtenidas del análisis de muestras de tejidos no tratados con antibióticos (blancos), a las cuales se adicionaron concentraciones conocidas de FFC y FFC-a en un rango entre 0.1 y $10 \mu \mathrm{g} / \mathrm{g}$. Las concentraciones plasmáticas y tisulares de FFC y FFC-a fueron determinadas de acuerdo a la técnica descrita por Hormazábal et al (20), con leves modificaciones - principalmente respecto a la cantidad de tejido utilizado. En este estudio, se utilizaron $2 \mathrm{~g}$ de tejido en el análisis de las muestras de ovinos. Para analizar las muestras de plasma el procedimiento fue similar pero se utilizó $1 \mathrm{~mL}$ de plasma. El mismo método de extracción fue utilizado en el análisis de las muestras de conejos, donde se utilizaron $0.5 \mathrm{~g}$ de tejido y $0.5 \mathrm{~mL}$ de plasma para determinar las concentraciones de FFC y FFC-a. 
La droga patrón y su metabolito fueron analizados utilizando una columna analítica Supelcosil LC18 (4.6 $\times 150 \mathrm{~mm}$; $5 \mu \mathrm{m}$; Supelco Inc., Bellefonte, PA, USA), mantenida en un horno de columna a $30^{\circ} \mathrm{C}$ (CTO-10AS vp; Shimadzu, Kyoto, Japan). Para FFC, la fase móvil fue fosfato de potasio $(0.01 \mathrm{M})$ y acetonitrilo $90: 10 \mathrm{v} / \mathrm{v}$, adicionando $100 \mu \mathrm{L}$ de trietilamina y $2 \mathrm{~mL}$ de dodecilo sulfato de sodio. El pH fue ajustado a 4.0. FFC y FFC-a fueron detectados a $225 \mathrm{~nm}$ mediante detector UV (SPD-10Avp-Detector, Shimadzu, Japan). Para FFC-a, la fase móvil fue una mezcla de dos soluciones: A y B (68:32). La solución A fue heptanosulfonato $0.02 \mathrm{M}$ y trisodio fosfato $0.025 \mathrm{M}$ ( $\mathrm{pH} 3.85)$. La solución $\mathrm{B}$ fue metanol conteniendo $0.1 \%$ trietilamina. La fase móvil fue bombeada a $0.8 \mathrm{~mL} / \mathrm{min}$ para FFC y a $0.6 \mathrm{~mL} /$ min para FFC-a.

Parámetros de validación de FFC y FFC-a. Los métodos analíticos para la determinación de FFC y FFC-a en ovinos y conejos fueron validados de acuerdo a los procedimientos descritos por la Unión Europea (21). El método analítico usado para la determinación de FFC y FFC-a en plasma de ovinos fue previamente validado y descrito por Palma et al (6). La validación de la metodología analítica en tejidos de ovinos fue previamente descrita en nuestro laboratorio por Pérez et al (22), y parámetros como linealidad $(\mathrm{r} 2)$, recuperación $(\%)$, precisión $(\mathrm{CV} \%)$, límite de detección (LOD) y límite de cuantificación (LOQ) fueron determinados. La validación del método analítico en plasma de conejos fue previamente descrito por Pérez et al (3). La metodología analítica en tejidos de conejos fue validada y entregó valores de recuperación promedio total de FFC y FFC-a de $82.22 \pm 5.7 \%$ y $81.72 \pm 3.8 \%$, respectivamente.

Análisis farmacocinético. Los parámetros farmacocinéticos de FFC y FFC-a fueron analizados utilizando un modelo no compartimental mediante el programa PK Solutions 2.0 (Summit Research Services, Ashland, OH, USA). La vida media de absorción ( $t_{1 / 2 a b}$ ) y vida media de distribución ( $t_{1 / 2 a}$ ) fueron calculados como In $2 /$ $k_{a b} y \ln 2 / a$, respectivamente, donde la tasa de absorción $\left(\mathrm{k}_{\mathrm{ab}}\right)$ es la constante de absorción de primer orden y a es la constante de distribución de primer orden. La concentración máxima $\left(\mathrm{C}_{\max }\right)$ y el tiempo en el cual se obtuvo $C_{\max }\left(T_{\max }\right)$ fueron calculados a partir de la curva concentración/ tiempo correspondiente a cada animal. El área bajo la curva de concentraciones plasmáticas en el tiempo fue calculada desde $t_{0}$ hasta el último tiempo de muestreo $\left(\mathrm{AUC}_{0-\mathrm{t}}\right)$ usando el método de los trapecios.
La proporción de metabolito fue calculada utilizando la fórmula:

$$
M R=\frac{A U C_{0 t} F F C-a}{A U C_{0_{-} t} F F C} \times 100
$$

Análisis estadístico. Los parámetros farmacocinéticos se expresan como promedio \pm EEM, y se compararon mediante la prueba $t$ de Student. Los promedios de concentraciones plasmáticas en el tiempo se analizaron mediante un análisis de varianza de dos vías. Se utilizó el Test de Bonferroni para la comparación de medias. Todos los análisis estadísticos fueron realizados utilizando el programa GraphPad Prism (v. 5.0; Graph Pad Software Inc., San Diego, CA, USA). El nivel de significancia se estableció en $p \leq 0.05$, y se consideró como tendencia valores de $p>0.05$ hasta $p<0.1$.

Aspectos éticos. Todos los experimentos fueron realizados con la autorización del Comité de Ética para la Experimentación Animal de la Facultad de Ciencias Veterinarias, Universidad de Concepción, Chile. El número de autorización del Comité de Ética fue CBE-12-2012. Los procedimientos experimentales fueron realizados de acuerdo con las recomendaciones del Reporte Final de la Comisión Europea, 2009 (Revisión de Directiva 86/609/EEC sobre la protección de animales utilizados para propósitos científicos) (22).

\section{RESULTADOS}

Análisis farmacocinético de FFC. Los promedios \pm EEM de las curvas de concentración en el tiempo de FFC se muestran en la figura 1. Las concentraciones plasmáticas de FFC fueron significativamente más altas en conejos que en ovinos $(p<0.01)$ a las $0.5,1,1.5$ y 2 horas posterior a la administración IM. Ambas especies alcanzaron las concentraciones máximas de FFC cerca de 1 hora posterior al tratamiento.

Los valores promedio de las variables farmacocinéticas calculadas para FFC se presentan en la tabla 1 . En conejos, el promedio de $C_{\max }$ y $A_{U} C_{0-t}$ de FFC fue mayor que en ovinos $(p<0.05)$. También se obtuvieron diferencias significativas al comparar $t_{1 / 2 a b}$ entre las dos especies, donde se observaron valores más altos en ovinos que en conejos. Por su parte, los valores promedio de $t_{1 / 2 a}$ mostraron una tendencia a ser más elevados en ovinos que en conejos. 


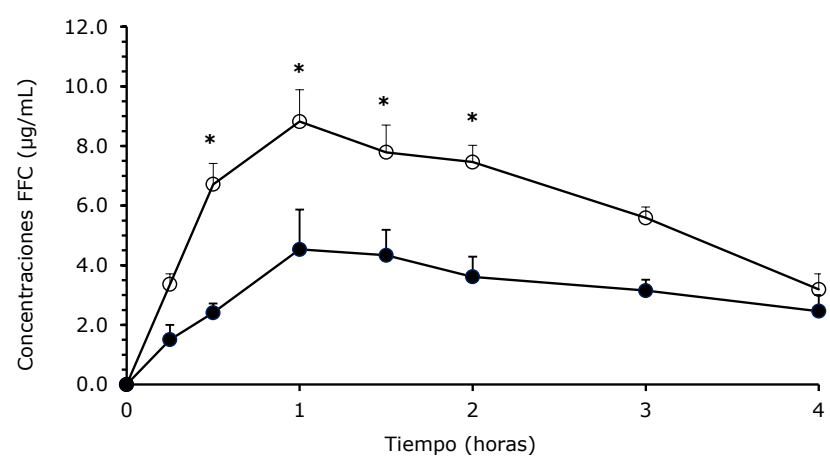

Figura 1. Curvas de concentraciones plasmáticas en el tiempo de florfenicol (FFC) administrado vía IM en dosis de $20 \mathrm{mg} / \mathrm{kg}$ en ovinos (círculos negros) $(n=5)$ y conejos (círculos blancos) $(n=6)$. Cada punto representa el promedio \pm EEM. * : Significancia $\mathrm{p}<0.05$, diferencias significativas entre ambas especies a la misma hora de muestreo.

Tabla 1. Valores promedio ( \pm EEM) de los parámetros farmacocinéticos de FFC en conejos y ovinos tratados con $20 \mathrm{mg} / \mathrm{kg}$ de FFC vía IM.

\begin{tabular}{lccc}
\hline \multicolumn{1}{c}{ Parámetro PK } & Ovinos & Conejos & Valor de $\mathbf{P}$ \\
\hline $\mathrm{C}_{\max }\left(\mu \mathrm{gL}^{-1}\right)$ & $4.94 \pm 1.25$ & $9.65 \pm 0.73$ & $0.008^{*}$ \\
$\mathrm{~T}_{\max }(\mathrm{h})$ & $1.60 \pm 0.37$ & $1.08 \pm 0.13$ & 0.187 \\
$\mathrm{~K}_{\mathrm{ab}}\left(\mathrm{h}^{-1}\right)$ & $1.17 \pm 0.16$ & $1.95 \pm 0.10$ & $0.002^{*}$ \\
$\mathrm{~T}_{1 / 2 \mathrm{ab}}(\mathrm{h})$ & $0.65 \pm 0.11$ & $0.36 \pm 0.02$ & $0.019^{*}$ \\
$\mathrm{~K}_{\mathrm{a}}\left(\mathrm{h}^{-1}\right)$ & $1.09 \pm 0.28$ & $2.03 \pm 0.07$ & $0.006^{*}$ \\
$\mathrm{~T}_{1 / 2 \mathrm{a}}(\mathrm{h})$ & $0.86 \pm 0.29$ & $0.35 \pm 0.01$ & 0.083 \\
$\mathrm{AUC}_{0-\mathrm{t}}\left(\mu \mathrm{g} \cdot \mathrm{h} \cdot \mathrm{mL}^{-1}\right)$ & $12.80 \pm 2.29$ & $24.42 \pm 1.22$ & $0.001^{*}$ \\
\hline
\end{tabular}

Cada valor representa el promedio \pm EEM para ovinos $(n=5)$ y conejos $(n=6)$. $C_{\max }$ : Concentración plasmática máxima; $T_{\max }$ : tiempo en obtener $\mathrm{C}_{\max } ; \mathrm{K}_{\mathrm{ab}}$ : constante de tasa de absorción; $\mathrm{t}_{1 / 2 \mathrm{ab}}$ : vida media de absorción; $\mathrm{K}_{\mathrm{a}}$ : constante de tasa de distribución; $t_{1 / 2 a}$ : vida media de distribución; $A_{U} C_{0-t}$ : área bajo la curva concentración-tiempo desde cero a la última concentración medida. *: Significancia $\mathrm{p}<0.05$, diferencias significativas entre ambas especies.

Concentraciones tisulares de FFC. Respecto a las concentraciones tisulares de FFC $(\mu \mathrm{g} / \mathrm{g})$, 4 horas posterior a su administración, estas fueron significativamente mayores $(p \leq 0.05)$ en conejos en comparación con los ovinos en todos los tejidos estudiados, a excepción de riñón. Sin embargo, hubo una tendencia en las concentraciones tisulares de FFC en riñón a ser mayores en conejos $\left(7.85 \pm 1.45 \mu \mathrm{g} \mathrm{g}^{-1}\right)$ en comparación a las concentraciones observadas en ovinos (4.74 $\left.\pm 0.55 \mu \mathrm{g} \mathrm{g}^{-1}\right)(\mathrm{p}=0.097)$ (Figura 2). En ambas especies, las mayores concentraciones de FFC fueron encontradas en riñón.

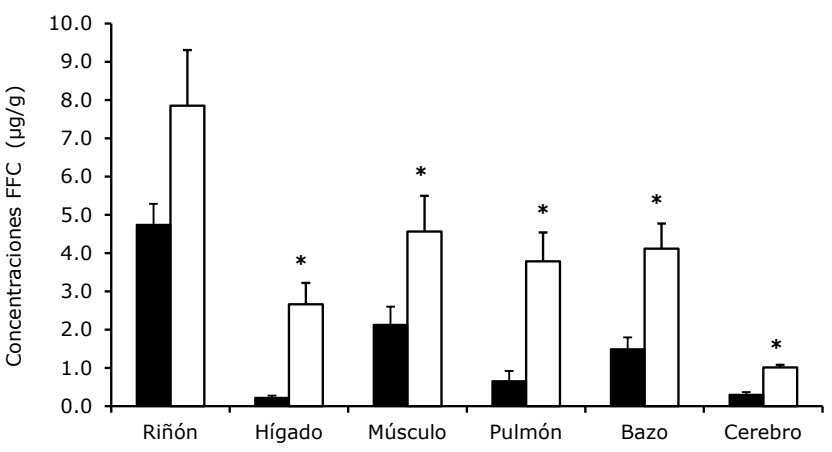

Figura 2. Promedios \pm EEM de concentraciones tisulares de florfenicol (FFC) a las 4 horas posterior a la administración IM en dosis de $20 \mathrm{mg} / \mathrm{kg}$ en ovinos (barras negras) $(n=5)$ y conejos (barras blancas) $(\mathrm{n}=6) . *$ : Significancia $\mathrm{p}<0.05$, diferencias significativas entre ambas especies.

Análisis farmacocinético de FFC-a. Se observaron concentraciones plasmáticas de FFC-a significativamente mayores en conejos respecto a los ovinos a las $1.5,2,3$ y 4 horas posterior a la administración de FFC $(p<0.01)$. Las curvas de concentraciones plasmáticas en el tiempo de FFC-a en ovinos y conejos se observan en la figura 3. FFC-a alcanzó valores de $C_{\max }$ y $A U C_{0-t}$ significativamente más altos en conejos que en ovinos, $(p<0.01)$ y $(p<0.01)$, respectivamente, como se observa en la tabla 2. En conejos, los valores calculados de proporción de metabolito fueron significativamente más altos $(12.70 \pm 3.07 \%)$ que los observados en ovinos $(3.99 \pm 0.87 \%)(p<0.05)$.

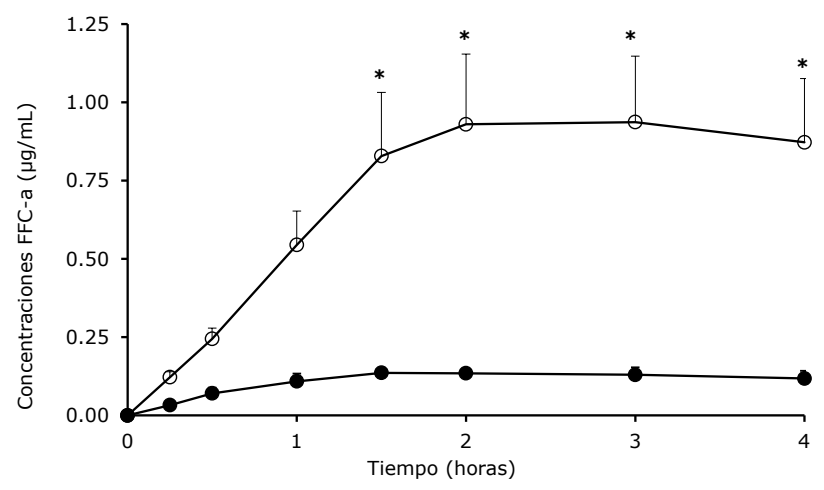

Figura 3. Curvas de concentraciones plasmáticas en el tiempo de florfenicol-amina (FFC-a) en ovinos (círculos negros) $(n=5)$ y conejos (círculos blancos) $(n=6)$ tratados con una dosis IM de $20 \mathrm{mg} / \mathrm{kg}$ florfenicol (FFC). Cada punto representa el promedio \pm EEM. *: Significancia $p<0.05$, diferencias significativas entre ambas especies a la misma hora de muestreo. 
Tabla 2. Valores promedio ( \pm EEM) de los parámetros farmacocinéticos de FFC-a en conejos y ovinos tratados con $20 \mathrm{mg} / \mathrm{kg}$ de FFC por vía IM.

\begin{tabular}{lccc}
\hline \multicolumn{1}{c}{ Parámetro PK } & Ovinos & Conejos & Valor de P \\
\hline $\mathrm{C}_{\max }(\mu \mathrm{g} \mathrm{mL}-1)$ & $0.17 \pm 0.01$ & $1.04 \pm 0.20$ & $0.004^{*}$ \\
$\mathrm{~T}_{\max }(\mathrm{h})$ & $2.20 \pm 0.56$ & $2.92 \pm 0.33$ & 0.278 \\
$\mathrm{~K}_{\mathrm{ma}}\left(\mathrm{h}^{-1}\right)$ & $2.10 \pm 0.83$ & $2.51 \pm 0.45$ & 0.659 \\
$\mathrm{t}_{1 / 2 \mathrm{ma}}(\mathrm{h})$ & $0.69 \pm 0.30$ & $0.32 \pm 0.05$ & 0.213 \\
$\mathrm{~K}_{\mathrm{a}}\left(\mathrm{h}^{-1}\right)$ & $1.12 \pm 0.55$ & $2.71 \pm 0.46$ & 0.052 \\
$\mathrm{t}_{1 / 2 \mathrm{a}}(\mathrm{h})$ & $3.49 \pm 1.85$ & $1.05 \pm 0.73$ & 0.221 \\
$\mathrm{AUC}_{0-\mathrm{t}}\left(\mu \mathrm{g} \cdot \mathrm{h} \cdot \mathrm{mL}^{-1}\right)$ & $0.44 \pm 0.02$ & $2.93 \pm 0.62$ & $0.006 *$ \\
\hline
\end{tabular}

Cada valor representa el promedio \pm EEM para ovinos $(n=5)$ y conejos $(n=6) . C_{\max }$ : Concentración plasmática máxima; $T_{\max }$ : tiempo en obtener $\mathrm{C}_{\max } ; \mathrm{K}_{\mathrm{ma}}$ : Constante de aparición de metabolito; $\mathrm{t}_{1 / 2 \mathrm{am}}$ : vida media de aparición de metabolito; $\mathrm{K}_{\mathrm{a}}$ : Constante de tasa de distribución; $\mathrm{t}_{1 / 2 \mathrm{a}}$ : vida media de distribución; $\mathrm{AUC}_{0-\mathrm{t}}$ : área bajo la curva concentración-tiempo desde cero a la última concentración medida. * : Significancia $\mathrm{p}<0.05$, diferencias significativas entre ambas especies.

Concentraciones tisulares de FFC-a. LaS concentraciones tisulares de FFC-a fueron significativamente mayores $(p<0.05)$ en conejos en comparación con los ovinos en todos los tejidos analizados. En ambas especies, la mayor concentración del metabolito fue observada en el riñón (Figura 4).

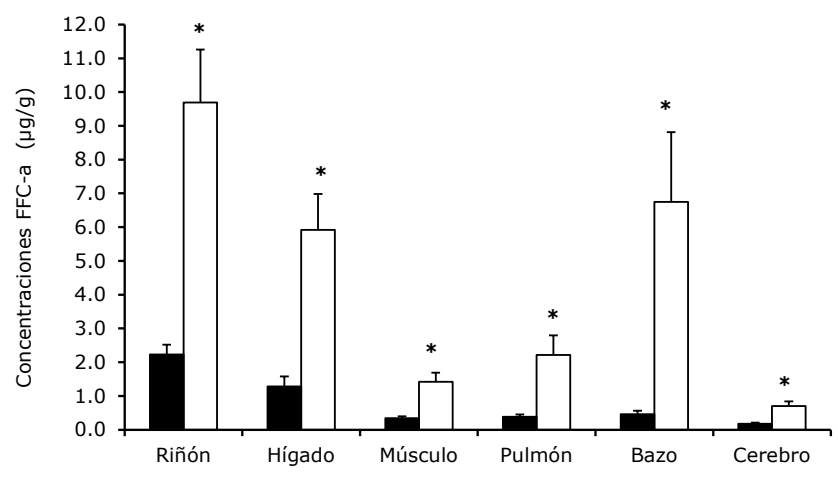

Figura 4. Promedios \pm EEM de concentraciones tisulares de florfenicol amina (FFC-a) en ovinos (barras negras) $(n=5)$ y conejos (barras blancas) $(n=6)$, a las 4 horas posterior a la administración IM de florfenicol (FFC) en dosis de $20 \mathrm{mg} /$ kg. *: Significancia $\mathrm{p}<0.05$, diferencias significativas entre ambas especies.

\section{DISCUSIÓN}

Este estudio comparó las concentraciones plasmáticas y tisulares de FFC y su metabolito FFC-a entre ovinos y conejos posterior a la administración IM de FFC. Los resultados demuestran que existen diferencias significativas entre estas especies en términos de la distribución del fármaco y su metabolito en plasma y tejidos, lo que indica diferencias de especie en los procesos farmacocinéticos. Se ha estudiado previamente la farmacocinética de FFC y FFC-a posterior a la administración IV de FFC en ovinos (10) y conejos (3). Posterior a la inyección IV de una dosis de FFC de $20 \mathrm{mg} / \mathrm{kg}$, las principales diferencias en la farmacocinética observadas entre estas dos especies se relacionaron con la vida media de eliminación y $\mathrm{AUC}_{0-\mathrm{t}}$ - las que fueron más elevadas en ovinos que en conejos. Más aún, el período de persistencia de las concentraciones plasmáticas sobre el LOQ en ovinos fue de 48 horas, mientras que en conejos fue de 8 horas. Por otro lado, el clearance plasmático total $\left(\mathrm{CL}_{T}\right)$ fue mayor en conejos que en ovinos, indicando que FFC es eliminado más rápidamente en los conejos que en los ovinos.

La farmacocinética de FFC posterior a la administración IM de $20 \mathrm{mg} / \mathrm{kg}$ también ha sido estudiada por Jianzhong et al (24) en ovinos. Estos autores reportaron valores mayores de AUC que los que observamos en el presente estudio - los que pueden ser explicados por su período de muestreo más largo (24 horas). Sin embargo, Jianzhong et al (24) observaron una vida media de absorción menor $(0.27 \pm 0.03$ horas) que los valores reportados en este estudio.

En conejos, Koc et al (9) obtuvieron un AUC posterior a la administración IM mayor que nuestros resultados de AUC (39.10 10.12 $\mu \mathrm{g} \cdot \mathrm{h} \cdot \mathrm{mL}^{-1}$ ) - sin embargo, esto es esperado ya que utilizaron una dosis más alta de FFC ( 25 $\mathrm{mg} / \mathrm{kg} \mathrm{PC}$ ). El-Aty et al (25) describieron valores de AUC para FFC $\left(86.56 \pm 11.99 \mu \mathrm{g} \cdot \mathrm{h} \cdot \mathrm{mL}^{-1}\right)$ que también son mayores que los que observamos en este estudio, posterior a la administración IM de una dosis de $30 \mathrm{mg} / \mathrm{kg}$ de FFC. Nuestros valores de $\mathrm{C}_{\max }$ de $\mathrm{FFC}$ fueron menores que los valores descritos por El-Aty et al $(21.65 \pm 2.57$ $\mu \mathrm{g} / \mathrm{mL}$ ) (25), pero mayores que el $\mathrm{C}_{\max }$ descrito por Koc et al $(8.65 \pm 2.19 \mu \mathrm{g} / \mathrm{mL})(9)$.

Como se muestra en la tabla 1 , los valores significativamente mayores del promedio de $\mathrm{C}_{\max }$ de FFC observados en conejos son consistentes con los valores mayores de $\mathrm{AUC}_{0-\mathrm{t}}$ para $\mathrm{FFC}$ 
en conejos en comparación con los ovinos, y son por lo tanto indicativos de una mayor biodisponibilidad del fármaco en conejos. En tanto el promedio de $t_{1 / 2 a b}$ fue significativamente menor en conejos que en ovinos, indicando una absorción más rápida en conejos.

Estas diferencias de absorción pueden ser causadas por diferencias en el flujo sanguíneo regional muscular entre estas especies (9), o pueden deberse a diferencias en la solubilidad dependiente de $\mathrm{pH}$ que afecta la absorción del fármaco (26). Además, la diferencia en las concentraciones de las soluciones de FFC que fueron administradas a los animales (solución de FFC al $30 \%$ en ovinos y solución de FFC al $6 \%$ en conejos) podrían explicar el alto nivel de absorción observado en conejos. La dilución de FFC usada en conejos puede haber influido en el proceso de absorción del fármaco, y haber facilitado una mayor absorción después de la inyección IM. No obstante, la dilución de FFC desde la formulación original fue necesaria con el fin de obtener un volumen de inyección apropiado y preciso en conejos ( $1 \mathrm{~mL}$ cada $3 \mathrm{~kg} \mathrm{PC}$ ).

Cabe destacar que la dilución de FFC que fue administrada en conejos estuvo compuesta por los tres mismos vehículos que la formulación original: (N-metil pirrolidona, propilenglicol y polietilenglicol). Debido a que estos vehículos estuvieron en la misma proporción en la dilución y en la formulación original, es probable que el proceso de liberación del fármaco haya sido similar.

Las concentraciones tisulares de FFC $(\mu \mathrm{g} / \mathrm{g})$ fueron más altas en conejos que en ovinos en todos los tejidos, aunque en riñón esta tendencia no fue estadísticamente significativa. Esto puede deberse a un mayor nivel de absorción de FFC en conejos, y también debido a diferencias en la distribución de FFC entre las dos especies.

Concentraciones plasmáticas significativamente más altas de FFC-a fueron observadas en conejos respecto a ovinos. Se encontraron marcadas diferencias en las curvas de concentración plasmática-tiempo entre ambas especies, indicando un incremento más rápido de la aparición de FFC-a en conejos. Además, los valores promedio de $\mathrm{C}_{\max }$ y $\mathrm{AUC}_{0-\mathrm{t}}$ para FFC-a fueron significativamente más elevados en conejos que en ovinos. Estos resultados indican que FFC experimenta un mayor nivel de metabolismo en conejos en comparación con los ovinos, y esto puede explicar las concentraciones plasmáticas más elevadas de FFC-a en esta especie. Esto se demuestra también mediante el cálculo de la proporción de metabolito, que fue mayor en conejos respecto a los ovinos.

Las concentraciones tisulares de FFC-a $(\mu \mathrm{g} / \mathrm{g})$ fueron significativamente más altas en conejos que en ovinos en todos los tejidos analizados. Esto puede estar relacionado con el mayor nivel de biotransformación observado de FFC, hacia su metabolito en conejos en comparación con los ovinos. También cabe mencionar que en el hígado de ambas especies, las concentraciones de FFC-a fueron mayores que aquellas de FFC, indicando que FFC es rápidamente metabolizado en el hígado. De acuerdo a lo esperado, en ambas especies, las mayores concentraciones de FFC y FFC-a se encontraron en tejido renal, ya que el riñón es el principal órgano de excreción para la droga madre y el metabolito (4).

El mayor nivel de metabolismo de FFC en conejos en comparación con los ovinos (medido a través de las concentraciones plasmáticas y tisulares de FFC-a) es probablemente indicativo de diferencias de especie en los sistemas enzimáticos que metabolizan fármacos, en particular el CYP450. Específicamente, podrían existir diferencias en la cantidad de enzimas, o diferencias en sus actividades, y/o subtipos especie-específicos de CYP450. Es sabido que existen diferencias en el sistema CYP450 entre distintas especies animales $(13,16)$. Respecto a subtipos específicos de CYP450, actividades del subtipo CYP3A han sido documentadas en conejos y ovinos (2).

Investigaciones previas han estudiado las diferencias en actividades de enzimas metabolizadoras de fármacos en preparaciones de hígado en varias especies de producción agrícola $(15,16)$. En el estudio de Dalvi et al (15), se encontraron diferencias significativas entre ovinos y conejos en la cantidad de CYP450 microsomal y en la actividad de benzfetamina $\mathrm{N}$-desmetilasa - con valores más altos en conejos. Considerando lo reportado por Liu et al (2), que mostró que CYP3A estuvo involucrado en el metabolismo de FFC en conejos, proponemos que las altas concentraciones de FFC-a observadas en esta especie son debido a un mayor nivel de actividad en su sistema enzimático microsomal hepático.

Desde un punto de vista terapéutico, el mayor nivel de metabolismo de FFC en conejos se relaciona con una menor persistencia de concentraciones plasmáticas antibacterianas 
útiles del fármaco. A pesar que se observaron concentraciones plasmáticas más elevadas de FFC en conejos respecto a los ovinos, el mayor nivel de metabolismo en conejos podría resultar en concentraciones plasmáticas terapéuticas de FFC menos persistentes, o que disminuyan más rápidamente.

Si tomamos en consideración un valor de concentración mínima inhibitoria (CMI) para FFC de $1 \mu \mathrm{g} / \mathrm{mL}$, como ha sido descrito para patógenos de ovinos (27), es posible observar que las concentraciones antibacterianas útiles de FFC persisten sobre este valor por un mayor tiempo en ovinos (aproximadamente 11 horas) que en conejos (aproximadamente 4 horas) - de acuerdo a los valores descritos por Jianzhong et al (24) y Park et al (1), respectivamente. Asimismo, después de la administración IV de FFC, Park et al (1) reportaron una menor duración de niveles plasmáticos de FFC en conejos, lo que se evidenció por una menor vida media de eliminación ( $0.90 \pm 0.20$ horas), que la descrita en ovinos (18.83 \pm 6.76 horas) (24).

Se han descrito valores de CMI de FFC para los principales patógenos en ovinos. Berge et al (27) describieron valores de CMI para $M$. haemolytica y para $P$. multocida que oscilaron entre 0.25 y $1 \mu \mathrm{g} / \mathrm{mL}$ (valores mínimos y máximos de CMI), desde aislados bacterianos obtenidos desde ovinos con enfermedad del tracto respiratorio. En el presente estudio, las concentraciones plasmáticas de FFC en ovinos estuvieron por encima de estos valores de CMI durante las 4 horas en que fueron medidas las concentraciones plasmáticas (las concentraciones de FFC oscilaron entre 1.5 y $4.5 \mu \mathrm{g} / \mathrm{mL}$ ). Por lo tanto, las concentraciones de FFC fueron 4 veces más altas que los valores máximos de CMI descritos para patógenos de ovinos. En conejos, Koc et al (9) han sugerido utilizar una CMI de $2 \mu \mathrm{g} / \mathrm{mL}$ como valor de referencia.

Aún no han sido reportados los valores de CMI desde aislados de los principales patógenos en conejos, pero en estudios de otras especies, una concentración de $2 \mu \mathrm{g} / \mathrm{mL}$ de FFC ha demostrado alta eficacia contra la mayoría de los patógenos (24). En nuestro estudio, las concentraciones plasmáticas de FFC estuvieron 4 veces por sobre este nivel durante las 4 horas de monitoreo de muestras.

Respecto a las concentraciones de FFC observadas en tejido pulmonar; en ovinos estas concentraciones $(0.66 \pm 0.27 \mu \mathrm{g} / \mathrm{mL})$ fueron mayores que los valores de $\mathrm{CMI}_{50}$ para $M$. haemolytica y $P$. multocida de 0.5 y $0.25 \mu \mathrm{g} / \mathrm{mL}$, respectivamente. En conejos, las concentraciones tisulares de pulmón $(3.79 \pm 0.75 \mu \mathrm{g} / \mathrm{g})$ fueron mayores que el valor de CMI de FFC de $2 \mu \mathrm{g} / \mathrm{mL}$. Estos resultados representan una importante contribución a información existente acerca de la eficacia terapéutica de FFC para el tratamiento de enfermedades causadas por patógenos respiratorios en ovinos y conejos, especialmente ya que los principales patógenos en ovinos como $P$. multocida también están presentes en conejos (28). Sin embargo, son necesarios futuros estudios para establecer la eficacia terapéutica de FFC en conejos, particularmente considerando el fenómeno de ingestión de cecotrofo que puede causar la recirculación de fármacos en esta especie $(13,29)$.

Los resultados del presente estudio muestran que el metabolismo de FFC en conejos es más alto que en ovinos, lo que se evidencia por mayores valores de $C_{\max }, T_{\max }$ y $A U C_{0-4 h}$ de FFC-a que fueron obtenidos en conejos. La eliminación de este fármaco puede que también sea más rápida en conejos $(1,3,10)$, de manera que se esperaría que las concentraciones plasmáticas de FFC decrecieran bajo los valores de CMI en más corto tiempo que en ovinos. Estos resultados demuestran que es importante considerar las diferencias de especie en la farmacocinética de fármacos al establecer esquemas terapéuticos de administración de fármacos para una especie animal determinada.

En conclusión, en este estudio se observaron diferencias significativas en la farmacocinética y concentraciones tisulares de FFC y su metabolito FFC-a entre conejos y ovinos. Esta información debería ser considerada cuando se establezcan las dosis y frecuencia de administración de este antibiótico en animales monogástricos (conejos) y rumiantes (ovinos). Estos resultados sugieren que puede ser necesaria una tasa de administración de FFC más frecuente en conejos debido a sus características farmacocinéticas.

\section{Conflictos de interés}

Los autores declaran no tener conflictos de interés.

\section{Agradecimientos}

Este estudio fue desarrollado con el apoyo de PFCHA/ BECASDOCTORADONACIONAL/2016-21160902 de CONICYT y Proyecto FONDECYT 1130473. 


\section{REFERENCIAS}

1. Park B-K, Lim J-H, Kim M-S, Hwang $\mathrm{Y}-\mathrm{H}$, Yun H-I. Pharmacokinetics of florfenicol and its major metabolite, florfenicol amine, in rabbits. J Vet Pharmacol Ther 2007; 30(1):32-36. DOI: https://doi.org/10.1111/ j.1365-2885.2007.00809.x

2. Liu N, Guo M, Mo F, Sun YH, Yuan Z, Cao $\mathrm{L}-\mathrm{H}$, et al Involvement of P-glycoprotein and cytochrome P450 3A in the metabolism of florfenicol of rabbits. J Vet Pharmacol Ther. 2011; 35(2):202-205. DOI: https://doi. org/10.1111/j.1365-2885.2011.01310.x

3. Pérez $R$, Palma $C$, Burgos $R$, Jeldres JA, Espinoza A, Peñailillo AK. The acute phase response induced by Escherichia coli lipopolysaccharide modifies the pharmacokinetics and metabolism of florfenicol in rabbits. J Vet Pharmacol Ther. 2015; 39(29:183-190. DOI: https://doi. org/10.1111/jvp.12244

4. Anadón $A$, Martínez MA, Martínez $M$, Ríos A, Caballero V, Ares I, et al. Plasma and tissue depletion of florfenicol and florfenicolamine in chickens. J Agric Food Chem. 2008; 56:11049-11056. DOI: https://doi. org/10.1021/jf802138y

5. Wang GY, Zheng $\mathrm{HH}$, Zhang $\mathrm{KY}$, Yang $F$, Kong $T$, Zhou B, et al. The roles of cytochrome P450 and P-glycoprotein in the pharmacokinetics of florfenicol in chickens. Iran J Vet Res. 2018; 19(1):9-14. http:// dx.doi.org/10.22099/ijvr.2018.4761

6. Palma C, Ramírez J, Benavente A, Cazanga $V$, Venegas M, Pérez R. Pharmacokinetics of florfenicol and florfenicol-amine after intravenous administration in sheep. J Vet Pharmacol Ther. 2012; 35(5):508-511. DOI: https://doi.org/10.1111/j.13652885.2011.01357.x

7. Embrechts J, Sedlák L, Hlavizna I, Hermanský $P$, Cechová I, Brozková $M$, et al The influence of the galenic form on pharmacokinetics of florfenicol after intramuscular administration in pigs. J Vet Pharmacol Ther. 2012; 36(1):92-94. DOI: https://doi.org/10.1111/ j.1365-2885.2012.01387.x

8. Park B-K, Lim J-H, Kim M-S, Hwang Y-H, Yun $\mathrm{H}-\mathrm{I}$. Pharmacokinetics of florfenicol and its metabolite, florfenicol amine, in dogs. Res Vet Sci. 2008; 84(1):85-89. DOI: https:// doi.org/10.1016/j.rvsc.2007.04.001
9. Koc F, Ozturk M, Kadioglu Y, Dogan E, Yanmaz LE, Okumus Z. Pharmacokinetics of florfenicol after intravenous and intramuscular administration in New Zealand White rabbits. Res Vet Sci. 2009; 87(1):102105. DOI: https://doi.org/10.1016/j. rvsc. 2008.10.010

10. Pérez $R$, Palma $C$, Drápela $C$, Sepúlveda $M$, Espinoza A, Peñailillo AK. Pharmacokinetics of florfenicol after intravenous administration in Escherichia coli lipopolysaccharide-induced endotoxaemic sheep. J Vet Pharmacol Ther. 2014; 38(2):144-149. DOI: https://doi. org/10.1111/jvp. 12160

11. Ramadan A, Abd El-Aty AM. Pharmacokinetics and distribution of florfenicol in bronchial secretions of healthy and Pasteurella multocida infected calves. Pharm Anal Acta. 2011; 2(1):117. DOI: https://doi. org/10.4172/2153-2435.1000117

12. Ruiz J, Zapata M, López C, Gutiérrez F. Florfenicol concentrations in milk of lactating cows postreated by intramuscular and intramammary routes. Rev MVZ Córdoba. 2010; 15(2):2041-2050. DOI: https://doi. org/10.21897/rmvz.314

13. Toutain $P-L$, Ferran $A$, Bousquet-Mélou $A$. Species differences in pharmacokinetics and pharmacodynamics. In: Cunningham F, Elliot J, Lees $P$, editors. Comparative and Veterinary Pharmacology, Handbook of Experimental Pharmacology. Berlin: Springer-Verlag; 2010. DOI: https://doi. org/10.1007/978-3-642-10324-7_ 2

14. Maté $M L$, Ballent $M$, Larsen $K$, Lifschitz $A$, Lanusse C, Virkel G. Gene expression and enzyme function of two cytochromes P450 $3 \mathrm{~A}$ isoenzymes in rat and cattle precision cut liver slices. Xenobiotica. 2015; 45(7):563570. DOI: https://doi.org/10.3109/004982 $\underline{54.2014 .1002122}$

15. Dalvi RR, Nunn VA, Juskevich J. Hepatic cytochrome P-450 dependent drug metabolizing activity in rats, rabbits and several food-producing species. J Vet Pharmacol Ther. 1987; 10:164-168. DOI : https://doi.org/10.1111/j.1365-2885.1987. tb00094.x 
16. Nebbia $C$, Dacasto $M$, Giaccherino AR, Albo AG, Carletti M. Comparative expression of liver cytochrome P450- dependent monooxygenases in the horse and in other agricultural and laboratory species. Vet $\mathrm{J}$. 2003; 165(1):53-64. DOI : https://doi. org/10.1016/S1090-0233(02)00174-0

17. Ali BH, Al-Qarawi AA, Hashaad M. Comparative plasma pharmacokinetics and tolerance of florfenicol following intramuscular and intravenous administration to camels, sheep and goats. Vet Res Commun. 2003; 27(6):475-483. https:// doi.org/10.1023/a:1025741724701

18. Ismail M, El-Kattan YA. Comparative pharmacokinetics of florfenicol in the chicken, pigeon and quail. Br Poult Sci. 2009; 50(1):144-149. DOI: https://doi. org/10.1080/00071660802613286

19. AVMA. AVMA guidelines for the euthanasia of animals: 2013 edition. Schaumburg: American Veterinary Medical Association; 2013. https://www.avma.org/KB/Policies/ Documents/euthanasia.pdf?fbclid=IwAR3f fStVgfUcSvHHKHbzCIEOVoVh8riNyIJ2HpqA JVxGF3NjaPc4-2AKO5Q

20. Hormazábal V, Steffenak I, Yndestad M. Simultaneous determination of residues of florfenicol and the metabolite florfenicol amine in fish tissues by high-performance liquid chromatography. J Chromatogr. 1993; 616(1):161-165. https://doi. org/10.1016/0378-4347(93)80484-I

21. EMEA. Guideline on validation of analytical procedures: methodology [Internet]. London, UK: Committee for Veterinary Medicinal Products/European Agency for the Evaluation of Medicinal Products; 1998. URL Available from: https://www.ema.europa.eu/en/ documents/scientific-guideline/vich-gl2validation-analytical-procedures-methodologystep-7-consensus-guideline en.pdf

22. Pérez-Fernández $R$, Cazanga $V$, Jeldres $J A$, Silva PP, Riquelme J, Quiroz F, et al Plasma and tissue disposition of florfenicol in Escherichia coli lipopolysaccharide-induced endotoxaemic sheep. Xenobiotica. 2016; 47(5):408-415. DOI: https://doi.org/10.1 $\underline{080 / 00498254.2016 .1195522}$
23. European Commission. Expert working group on severity classification of scientific procedures performed on animals: Final Report. [Internet]. Brussels: European Commission; 2009. URL Available from: http://ec.europa.eu/environment/ chemicals/lab animals/pdf/report ewg.pdf

24. Jianzhong S, Xiubo L, Haiyang J, Walter $\mathrm{HH}$. Bioavailability and pharmacokinetics of florfenicol in healthy sheep. J Vet Pharmacol Ther. 2004; 27(3):163-168. DOI: https://doi.org/10.1111/j.13652885.2004.00574.x

25. El-Aty AM, Goudah A, El-Sooud KA, El-Zorba HY, Shimoda M, Zhou HH. Pharmacokinetics and bioavailability of florfenicol following intravenous, intramuscular and oral administration in rabbits. Vet Res Commun. 2004; 28(6):515-524. https://doi. org/10.1023/b:verc.0000040241.06642.49

26. Lin JH. Species similarities and differences in pharmacokinetics. Drug Metab Dispos. 1995; 23(10):1008-1021. https://www. ncbi.nlm.nih.gov/pubmed/8654187

27. Berge $A C$, Sischo WM, Craigmill AL. Antimicrobial susceptibility patterns of respiratory tract pathogens from sheep and goats. J Am Vet Med Assoc. 2006; 229(8):1279-1281. DOI: https://doi. org/10.2460/javma.229.8.1279

28. Stahel ABJ, Hoop RK, Kuhnert P, Korczak BM. Phenotypic and genetic characterization of Pasteurella multocida and related isolates from rabbits in Switzerland. J Vet Diagn Invest. 2009; 21(6):793-802. DOI: https:// doi.org/10.1177/104063870902100605

29. Meredith $A L$, Prebble JL. Impact of diet on faecal output and caecotroph consumption in rabbits. J Small Anim Pract. 2017; 58(3):139-145. DOI: https://doi. org/10.1111/jsap. 12620 